\title{
DNA specificity determinants of Escherichia coli tryptophan repressor binding
}

\author{
Steven Bass, ${ }^{1}$ Paul Sugiono, ${ }^{1}$ Dennis N. Arvidson, ${ }^{2}$ Robert P. Gunsalus, ${ }^{2}$ and Philip Youderian ${ }^{1}$ \\ ${ }^{1}$ Department of Biological Sciences, University of Southern California, Los Angeles, California 90089-1481 USA; ${ }^{2}$ Department \\ of Microbiology and the Molecular Biology Institute, University of California-Los Angeles, Los Angeles, California 90024 USA
}

\begin{abstract}
We have analyzed the sequence-specific interaction between the Escherichia coli tryptophan (Trp) repressor and its operator using challenge phage vectors. These phages, derivatives of Salmonella phage P22 that have substitutions of synthetic, symmetric trp operators for the P22 mnt operator, provide a genetic assay for DNA binding in vivo. Phages carrying operators that retain the determinants of Trp repressor binding efficiently lysogenize cells producing repressor; in contrast, phages with operators missing critical determinants kill such hosts. The binding determinants revealed by this assay corroborate a simple docking model for the Trp repressor-operator interaction postulated from the repressor crystal structure, and account for both the specificity of repressor binding and the ability of Trp repressor to recognize multiple, tandem DNA sites.
\end{abstract}

[Key Words: Trp repressor; DNA binding; trp operator; phage P22]

Received February 23, 1987; revised version accepted June 6, 1987.

The Escherichia coli trpR gene encodes a trans-acting negative regulator of tryptophan biosynthesis /Cohen and Jacob 1959; Yanofsky 1971; Zubay et al. 1972). The product of the $\operatorname{trp} R$ gene is a small peptide of 108 amino acid residues that assembles as a dimer aporepressor molecule and binds the corepressor, L-tryptophan, to form an active repressor complex (Gunsalus and Yanofsky 1980; Joachimiak et al. 1983; Arvidson et al. 1986). Active Trp repressor binds specifically to at least three targets on the E. coli chromosome to regulate initiation of transcription of the trp operon (Bennett and Yanofsky 1978), the $a r o H$ gene (encoding the tryptophan-repressible 3-deoxy-D-arabino heptulosonic acid-7-phosphate synthetase) (Zurawski et al. 1981; Grove and Gunsalus 1987), and the $\operatorname{trp} R$ gene itself (Gunsalus and Yanofsky 1980). The three operators share extensive sequence homology and are located at different positions relative to their respective promoters (Fig. 1).

The trp and $a r o H$ operators are composed of multiple, tandem Trp repressor binding sites, presumably each of which is bound by a repressor dimer. The trp operator has three tandem sites, whereas the $a r o H$ operator has two sites. The $\operatorname{trpR}$ operator is the simplest of the three and is presumably bound by a single repressor dimer (Kumamoto et al. 1987). An axis of twofold symmetry divides each of the six natural binding sites into two half-sites. A consensus of the 12 half-sites shows that the 10 central base pairs of each half-site are strongly conserved. Constitutive mutations in the operator controlling trp operon expression identify three of these 10 base pairs as critical for Trp repressor binding (Bennett and Yanofsky 1978).

As a first step in identifying the nucleotide base pair/ amino acid residue interactions that underlie the binding of Trp repressor to DNA, we have made all possible symmetric pairs of single base pair substitutions at each position of a Trp repressor binding site and have assayed the binding of wild-type Trp repressor to each of these sites in vivo. To assay Trp repressor binding, we have constructed derivatives of phage P22, called challenge phages, that place the expression of the P22 ant (antirepressor) gene under the control of each variant repressor binding site.

A challenge phage selectively lysogenizes, and spares from death, host cells producing an activity that binds the substituted operator site. This occurs because the "decision" between lytic and lysogenic development made by a challenge phage is governed by the state of occupancy of the operator controlling transcription of the ant gene. Substitution of a synthetic, idealized trp operator for the naturally occurring P22 (mnt) operator places ant expression under the control of $\operatorname{Trp}$ repressor. After infection of a sensitive sup ${ }^{0}$ host, a challenge phage will establish lysogeny if its operator is bound by Trp repressor, and confer a kanamycin-resistant phenotype upon its host. Alternatively, if the operator controlling ant synthesis is free of repressor, a challenge phage will synthesize antirepressor, grow lytically, and efficiently kill its host (Benson et al. 1986).

We have used a set of challenge phages carrying 


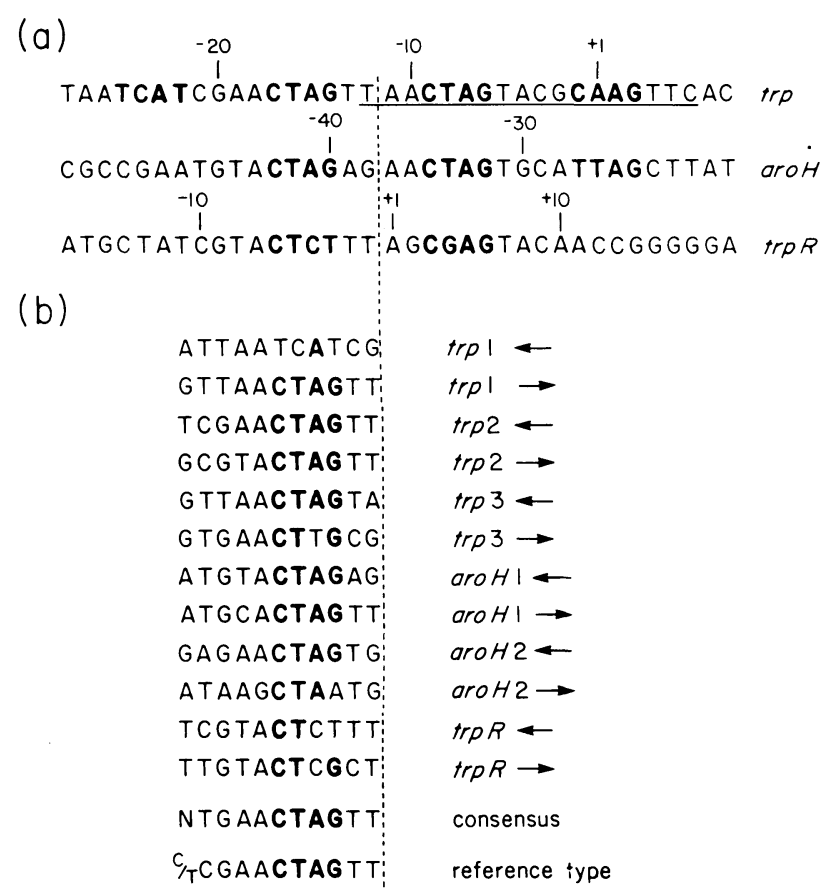

Figure 1. Trp repressor binds to multiple targets. (a) The DNA sequences of the top strands of the three natural operators controlling the E. coli trp, $\operatorname{aroH}$, and $\operatorname{trpR}$ operons are shown; numbers indicate the positions of base pairs with respect to the various start points of transcription (see Gunsalus and Yanofsky 1980). The most critical base pairs for repressor binding in each operator half-site are indicated in boldface. Presumably, three Trp repressor dimers contact successive pairs of major grooves of the trp operator, and two dimers bind the $a r o H$ operator, adjacent to faces of the double helix that are rotated with respect to one another by about $270^{\circ}$ (Kumamoto et al. 1987). (b) Each half-site of the six repressor binding sites is indicated by an arrow. Base pairs are included in the consensus sequence if they occur in 6 or more of the 12 half-sites, otherwise a position is designated " $\mathrm{N}$ ". The reference-type sequence is the synthetic trp operator sequence used in these studies.

variant trp operators derived from reference-type trp operators by symmetric single base pair substitutions to define the base pairs within the trp operator that are critical for Trp repressor binding. Our results, taken together with the crystal structure of Trp repressor (Schevitz et al. 1985) and data from chemical protection experiments that define the interaction of Trp repressor with naturally occurring operators (Kumamoto et al. 1987), enable us to construct a model for the specificity determinants of the Trp repressor/operator interaction.

\section{Results}

\section{A selection for Trp repressor binding}

To begin to analyze the Trp repressor-operator binding interaction, we synthesized a symmetric trp operator (Fig. 1). This oligonucleotide was introduced into the subcloned regulatory region of the phage P22 ant operon on plasmid vector pPY140 to construct plasmid pPY360. As shown in Figure 2, this idealized, symmetric Trp re- pressor binding site (designated "ref2" in Table 1) is substituted for the mnt operator, and is located at the start point of ant transcription. Plasmid pPY360 was crossed with $\mathrm{P} 22 \mathrm{Kn} 9$ arc-amH1605 phage to construct a challenge phage carrying the synthetic trp operator in place of the mnt operator (Benson et al. 1986). This phage cannot lysogenize a sensitive $\sup p^{0} \operatorname{tr} p R^{+}$host, indicating that the amount of Trp repressor synthesized from the Salmonella chromosome is insufficient to effect full repression of the phage-borne ant operon. Because the fractional occupancy of a DNA-binding site increases with increasing repressor concentration, we reasoned that the challenge phage with this idealized trp operator should lysogenize a host that overproduces Trp repressor and can saturate this site.

To construct such a host, we transformed a derivative of plasmid pRPG47 (Arvidson et al. 1986), pPY150, into the sup ${ }^{0}$ Salmonella host MS1868/F' lacI ${ }^{\mathrm{Q} 1}$. Plasmid pPY150 (Benson et al. 1986) carries the cloned E. coli $\operatorname{trpR}$ gene under the control of the inducible lacUV5 promoter; the $\mathrm{F}^{\prime}$ episome carried by the host overproduces Lac repressor and prevents transcription of the $\operatorname{trp} R$ gene from pPY150 in the absence of inducer. This host allows us to regulate the amount of Trp aporepressor produced from plasmid pPY150 by varying the amount of added inducer of $\mathrm{Lac}$ repressor, isopropyl- $\beta$-D-thiogalactoside (IPTG).

As shown in Figure 3(a), MS1868/F' lacl ${ }^{\mathrm{Q} 1}$ carrying plasmid pPY150 is efficiently lysogenized, in an IPTGdependent way, by a challenge phage with an idealized trp operator. The efficiency of lysogeny increases over 1000 -fold upon induction of $\operatorname{tr} p R$ transcription from the lac promoter with IPTG in the presence of excess corepressor. Therefore lysogeny is dependent upon expression of $t r p R$ from pPY 150 .

The efficiency of lysogeny is also dependent on the amount of exogenous corepressor. As shown in Figure 3 (b), lysogeny is inefficient at low tryptophan concentrations and increases to about $50 \%$ at higher tryptophan concentrations. These data show that the amount of active repressor complex is limited by the amount of added corepressor. At low concentrations of IPTG or tryptophan, lysogens grow as small, dark green colonies on green indicator plates. This phenotype indicates that these lysogens are unstable and produce insufficient Trp repressor activity to maintain stable repression of the ant gene. At high IPTG and tryptophan concentrations, lysogens form large, white colonies.

A similar bacterial host, MS1868/F' $1 a c I^{\mathrm{Q} 1}$ carrying pPY158, a plasmid that is missing $\operatorname{trp} R$ but is otherwise isogenic with pPY150, is efficiently killed by the same challenge phage, even at high levels of IPTG and tryptophan. Therefore, the ability of a bacterial host to survive infection by a challenge phage with the trp operator depends solely on the plasmid-borne $\operatorname{trp} R$ gene.

\section{Construction of Trp challenge phages}

Thirty variants of two idealized, symmetric trp operators were synthesized and cloned into a unique restriction site of a plasmid carrying the subcloned P22 ant 


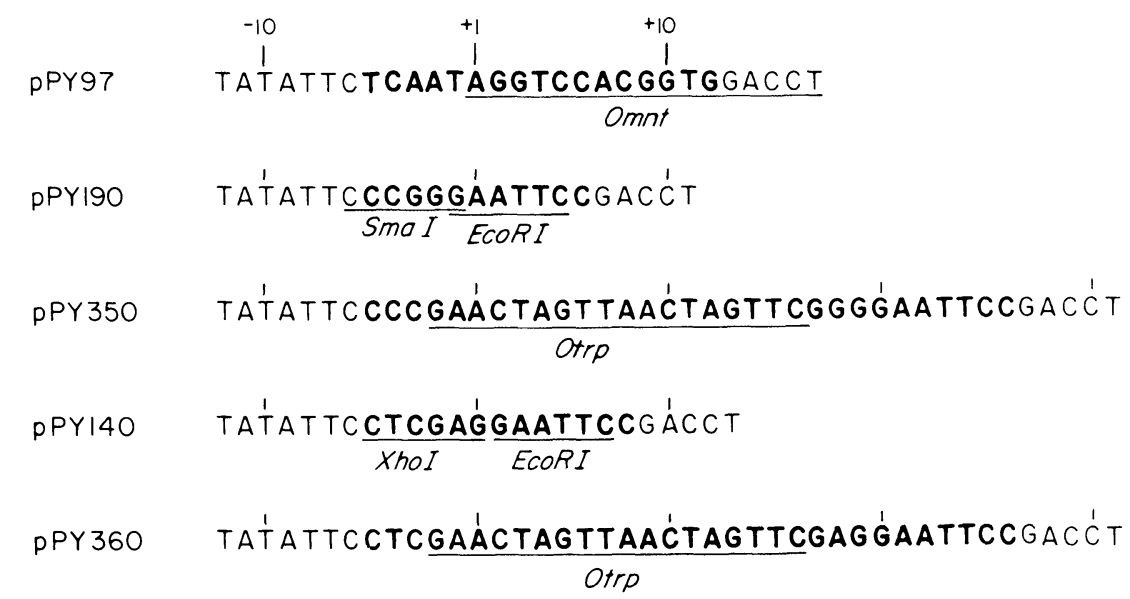

Figure 2. $\operatorname{trp}$ operators are positioned at the start point of ant transcription on challenge phages. The positions of the wild-type mnt operator on plasmid pPY97, of substitutions for the mnt operator on plasmids pPY190 and pPY140, and of the synthetic, reference trp operators in these plasmids are shown with respect to the start point of transcription of the ant promoter. The subclone of the trp operator (designated ref1 in Table 1) in pPY190 is pPY350; the subclone (of ref2) in pPY140 is pPY360. Operators were cloned into the SmaI site of pPY190 as 18-mers, or the filled-in XhoI site of pPY140 as 14-mers. pPY350 and pPY360 differ by transitions at positions -4 and +18 ; these differences have no apparent effect on repressor binding. Differences between the operator regions of these plasmids are indicated in boldface.

control region (either pPY140 or pPY190; Fig. 2). Each of these variant operators differs from one of the two idealized trp operators by a pair of symmetric single base pair substitutions (Table 1). Plasmids with cloned operators were transformed into Salmonella, and the transformants were infected with $\mathrm{P} 22 \mathrm{Kn} 9$ arc-amH1605 phage to cross these operators onto phage. Progeny phage that had acquired operator substitutions were selected as virulent recombinants on the host MS1582 (P22 $\left.\mathrm{c2}^{+} \mathrm{mnt}^{+}\right)$ (see Benson et al. 1986). Twenty-seven of 31 operatorcontaining plasmids yielded recombinants having a virulent phenotype. The genotypes of these recombinant phages were confirmed by the detection of restriction fragment length polymorphisms corresponding to each of their genetic markers and by the DNA sequence analysis of each operator on phage DNA (see Table 1).

\section{Operator determinants of Trp repressor binding}

Table 1 shows the efficiencies of survival of Trp repressor-producing cells infected with each of the series of challenge phages carrying symmetrically altered trp operators in the presence of an excess of corepressor. Efficiencies of survival range from $50 \%$ for phages carrying reference $\operatorname{tr} p$ operators, to less than $2 \times 10^{-6}$ percent for phages carrying a subset of mutant operators. These Trp repressor-producing cells are efficiently lysogenized by phages with variant operators that retain critical contacts for repressor binding, but not by phages with operators that lack critical contacts. Figure 4 summarizes these data.

Base-pair substitutions at four positions $( \pm 6, \pm 5, \pm 4$, and \pm 3 ) within the operator half-site reduce the efficiency of survival of the infected host by more than 1000-fold, and identify these four base pairs as the most critical determinants of Trp repressor binding. A C: G base pair at position \pm 6 in the half-site is absolutely required; challenge phages carrying operators with any other nucleotide base pair at this position evoke cell death. Phages carrying $\mathrm{T}: \mathrm{A}$ or $\mathrm{C}: \mathrm{G}$ base pairs at position \pm 5 of the operator lysogenize cells with nearly equal, high efficiencies, whereas phages with $\mathrm{A}: \mathrm{T}$ or $\mathrm{G}: \mathrm{C}$ changes at this position do not. Phages carrying operators with $\mathrm{G}: \mathrm{C}$ or $\mathrm{T}: \mathrm{A}$ base pairs at position \pm 4 , or $A$ : $T$ base pairs at position \pm 3 , also kill their hosts.

As we have shown above (Fig. 3), when challenge phage infections are carried out under conditions of low exogenous corepressor concentrations, the efficiency of cell survival is low, presumably because there is less active Trp repressor complex in the infected cells. We see more subtle effects of the operator substitutions by measuring efficiencies of survival after challenge at different concentrations of corepressor. Table 1 shows that operators that bind repressor efficiently in the presence of high concentrations of tryptophan may be subdivided further into three classes: operators bound at the lowest concentration of added tryptophan, operators that are not bound at the lowest concentration but are bound at an intermediate concentration, and operators bound only at the highest concentration.

\section{Conclusions}

Operator determinants of binding

Previously, we showed that the E. coli Trp repressor can function to repress transcription of the P22 ant operon when a symmetric trp operator site is substituted for the phage mnt operator at the start point of ant transcription. P22 challenge phages with an idealized, symmetric trp operator lysogenize host cells that produce sufficient Trp repressor activity and lyse cells that do not produce sufficient Trp repressor activity (Benson et al. 1986). 
Table 1. DNA-binding properties of synthetic trp operators

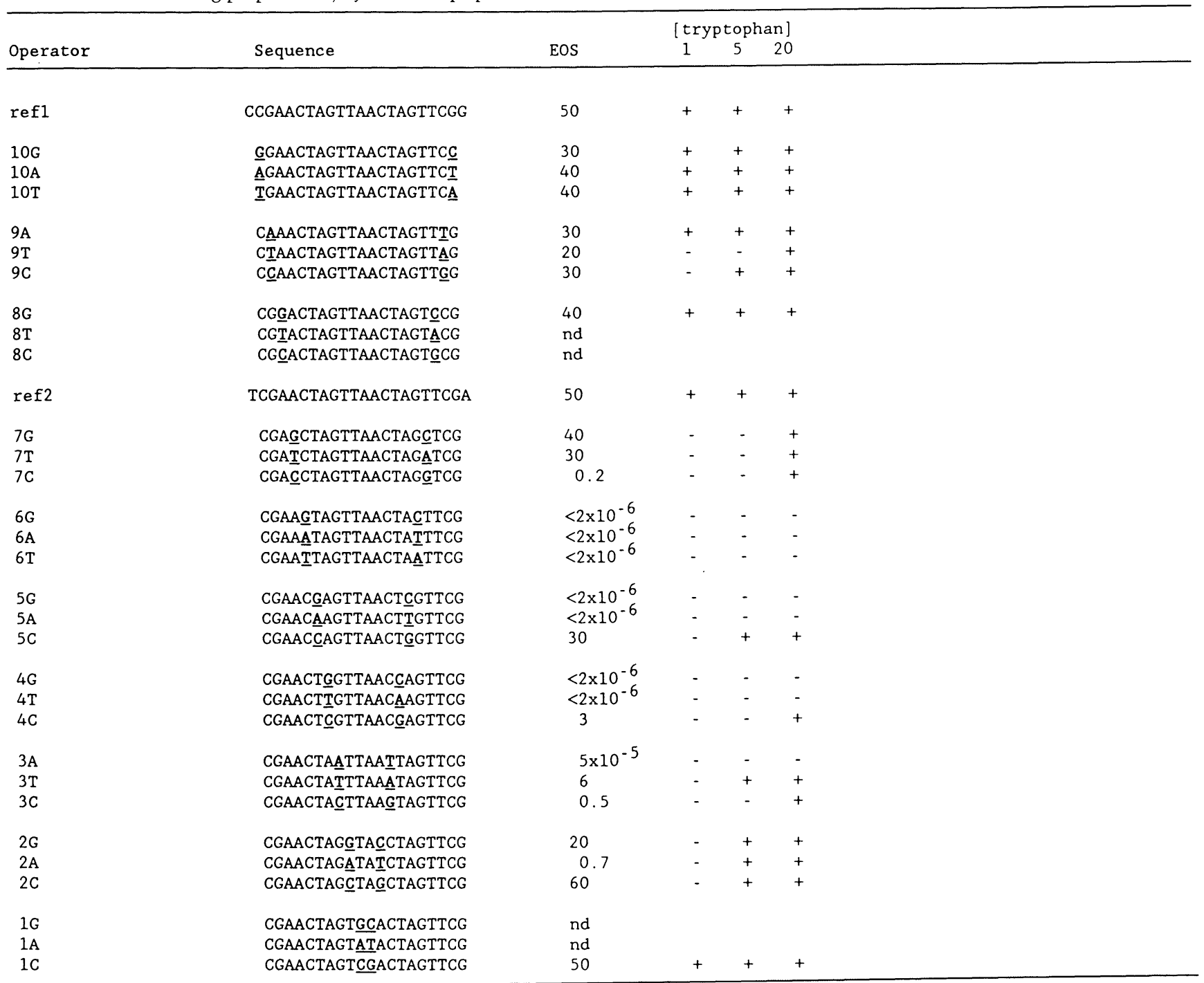

Symmetric operators carried by the $\mathrm{P} 22 \mathrm{Kn} 9$ arc-am H1605 challenge phages are indicated as either reference operators (refl and ref2) or variant operators. Variant operators carry symmetric substitutions (underlined), and are designated by number and variant nucleotide in the top strand of the left operator half-site (e.g., 10G). Positions in the operator half-site are numbered outward from the center of symmetry. To determine the efficiency of survival (EOS) of host MS1868/F'lacI ${ }^{\mathrm{Q} 1}$ (pPY150) infected with a particular challenge phage, cells were first grown overnight in LB medium at $37^{\circ} \mathrm{C}$. The overnight culture was diluted 100 -fold into LB medium with ampicillin $(50 \mu \mathrm{g} / \mathrm{ml})$ and IPTG $\left(10^{-5} \mathrm{M}\right)$, and grown to a density of $4 \times 10^{8} / \mathrm{ml}$ at $37^{\circ} \mathrm{C}$. Phage were added to a final multiplicity of infection of 25 , and allowed to adsorb for $20 \mathrm{~min}$ at $25^{\circ} \mathrm{C}$. Infected cells were diluted and spread on green tryptophan drop-out plates supplemented with ampicillin, kanamycin, tryptophan $(40 \mu \mathrm{g} / \mathrm{ml})$, and IPTG $\left(10^{-5} \mathrm{M}\right)$. The efficiency of survival is the percent of the titer of cells assayed under these plating conditions divided by the titer of input cells assayed on green plates with ampicillin. At high IPTG and tryptophan concentrations, the efficiency of survival of cells carrying the Trp repressor-producing plasmid is independent of the multiplicity of the challenge phage infection for multiplicities between 10 phage/cell and 100 phage/cell (data not presented). The efficiencies of survival were not determined (nd) for phages carrying operators $8 \mathrm{~T}, 8 \mathrm{C}, 1 \mathrm{G}$, and $1 \mathrm{~A}$, since they lysogenize a host that fails to produce Trp repressor, and these operators are presumably bound by a second endogenous Salmonella repressor (unpubl.). Under the same conditions, all but these four phages fail to lysogenize a host carrying plasmid pPY158, an otherwise isogenic plasmid that fails to produce Trp repressor. In a separate set of experiments, cells infected with phage were spread on green trpyptophan drop-out plates containing ampicillin, kanamycin, IPTG $\left(10^{-5} \mathrm{M}\right)$, and either 1,5 , or $20 \mu \mathrm{g} / \mathrm{ml}$ tryptophan. The efficiencies of survival of these "challenges" are scored either as $+\left(>10^{-2}\right)$ or $-\left(<10^{-2}\right)$. DNAs from the phage stocks used in these experiments were sequenced to confirm their operator genotypes.

To understand the basis of sequence-specific operator recognition by Trp repressor in vivo, we synthesized an exhaustive series of trp operators and recombined them onto challenge phage vectors. Each trp challenge phage contains a variant operator that differs from an otherwise isogenic reference operator by a pair of symmetric 

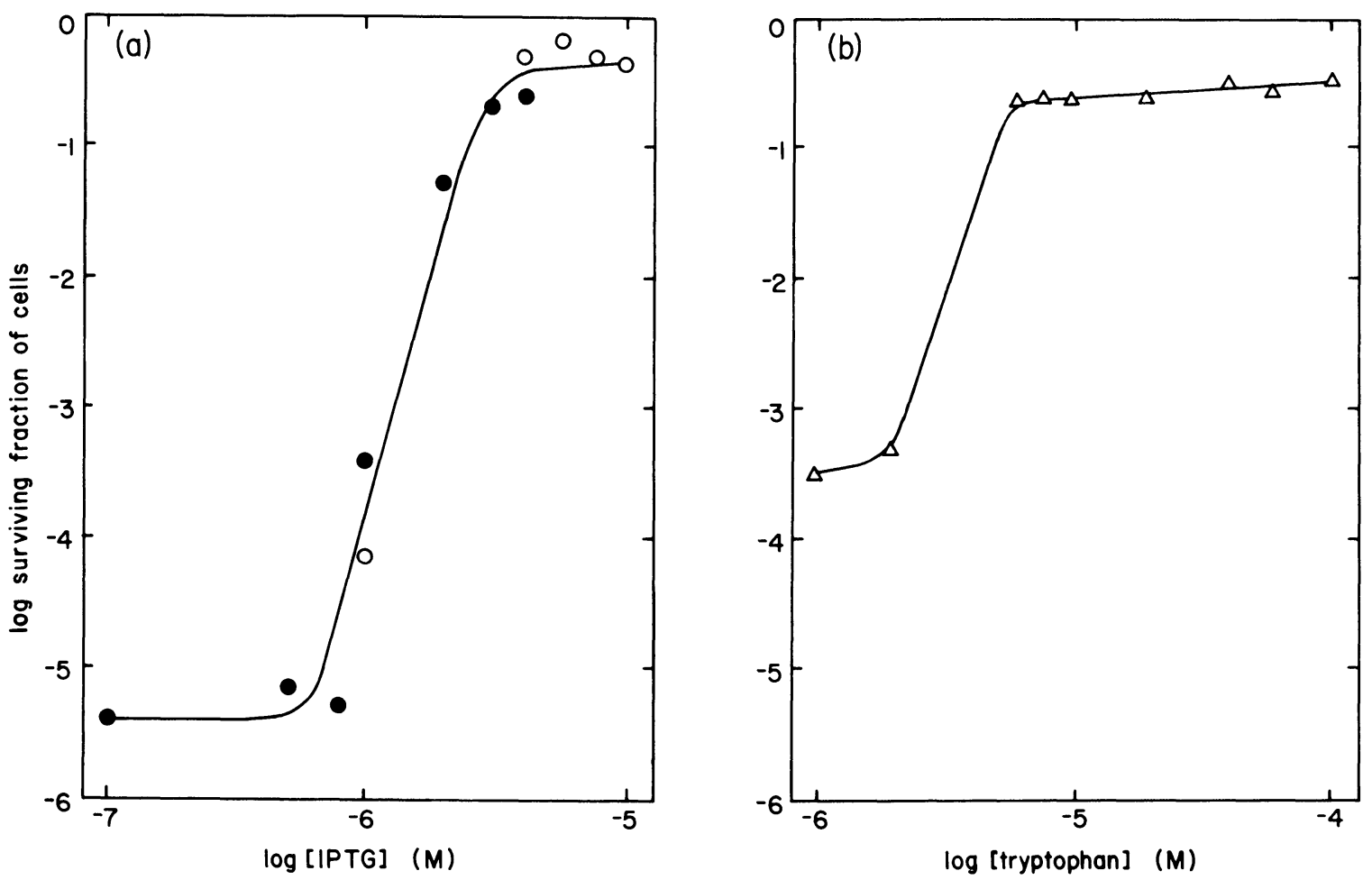

Figure 3. Trp repressor binding to a synthetic trp operator depends upon the levels of both aporepressor and corepressor. To determine the dependence of the efficiency of survival upon IPTG concentration, a fresh overnight culture of MS1868/F'lacIQ1 (pPY150) was diluted 100 -fold into LB medium with ampicillin $(50 \mu \mathrm{g} / \mathrm{ml})$ and IPTG at the indicated concentrations, and grown to a density of $4 \times 10^{8} / \mathrm{ml}$ at $37^{\circ} \mathrm{C}$. Phage with the reference-type operator ref2 were added to a final multiplicity of infection of 25 , and allowed to adsorb for $20 \mathrm{~min}$ at $25^{\circ} \mathrm{C}$. Infected cells were diluted and spread on green tryptophan drop-out plates supplemented with ampicillin, kanamycin, tryptophan $(40 \mu \mathrm{g} / \mathrm{ml})$, and the indicated concentrations of IPTG. The efficiency of survival is the percent of the titer of cells assayed under these plating conditions divided by the titer of input cells assayed on green plates with ampicillin. To determine the dependence of the efficiency of survival upon tryptophan concentration, MS1868/F'lacI ${ }^{\mathrm{Q} 1}$ (pPY150) was diluted 100-fold into LB medium with ampicillin $(50 \mu \mathrm{g} / \mathrm{ml})$ and IPTG $\left(10^{-5} \mathrm{M}\right)$, grown to a density of $4 \times 10^{8} / \mathrm{ml}$ at $37^{\circ} \mathrm{C}$, and infected with phage at a final multiplicity of 25 . After adsorption for $20 \mathrm{~min}$ at $25^{\circ} \mathrm{C}$, infected cells were diluted and spread on green tryptophan drop-out plates supplemented with ampicillin, kanamycin, IPTG $\left(10^{-5} \mathrm{M}\right)$, and the indicated concentrations of tryptophan.

single base pair substitutions. Phages carrying variant operators that retain the nucleotide base pairs required for Trp repressor binding efficiently lysogenize cells producing sufficient Trp repressor, whereas phages carrying variant $\operatorname{tr} p$ operators lacking critical contacts for Trp repressor binding lyse the same host. By comparing the efficiencies of survival of Trp repressor-producing cells after infection with each of this series of Trp challenge phages, we have identified the operator determinants of the sequence-specific binding of Trp repressor.

The idealized $\operatorname{trp}$ operator extends $9 \mathrm{bp}$ in either direction from its axis of dyad symmetry, since mutations at base pair \pm 9 in both half-sites have significant effects on Trp repressor binding at low concentrations of added tryptophan, whereas mutations in the two flanking base pairs (positions \pm 11 and \pm 10 ) do not affect the ability of repressor to bind (Table 1). The most critical determinants of DNA recognition reside in four base pairs within each $\operatorname{trp}$ operator half-site, separated by four central base pairs. Phages carrying operators with nucleotide substitutions at these critical positions fail to lysogenize (Fig. 4). Consistent with these results, Bennett and Yanofsky (1978) have isolated constitutive muta- tions at positions $+4, \pm 5$, and -6 within the trp operator half-sites that represent four of these eight most critical changes. The $\mathrm{G}: \mathrm{C}$ base pairs in the natural operators implicated in binding by Kumamoto et al. (1987) include the same $\mathrm{G}$ : $\mathrm{C}$ base pairs identified as important by our genetic method.

\section{A model for the Trp repressor-operator interaction}

The active form of Trp repressor is an interlocked symmetric dimer (Joachimiak et al. 1983; Schevitz et al. 1985; Arvidson et al. 1986). Exposed on one face of the dimer are two symmetrically disposed "helix-turnhelix" secondary structures (one for each monomer) characteristic of many specific DNA-binding proteins (Pabo and Sauer 1984; Kelley and Yanofsky 1985; Schevitz et al. 1985). Consistent with the idea that this "helix-turn-helix" substructure of the Trp repressor protein is involved in DNA binding, the majority of dominant negative mutations in the $\operatorname{trp} R$ gene are located within the coding sequence corresponding to this region of the protein (Kelley and Yanofsky 1985; S. Bass, unpubl.).

A preliminary model for the binding of Trp repressor 


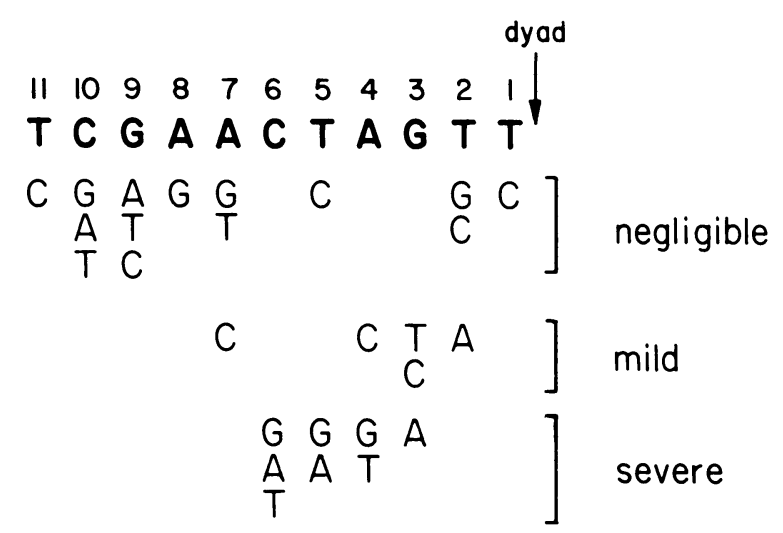

Figure 4. A subset of operator mutations interfere with Trp repressor binding. The data from experiments at high corepressor concentration presented numerically in Table 1 are represented in two dimensions. The idealized operator sequence occurs near the top of the diagram, numbered by position from the axis of symmetry (arrow). Immediately below the reference sequence are listed the changes that have negligible effects on repressor binding. Below the reference sequence are listed the changes that reduce the ability of repressor to bind. These changes are grouped into two categories, symmetric mutations that reduce survival 5- to 100 -fold (mild) and those that reduce survival more than 1000 -fold (severe).

to DNA may be constructed by docking this conserved secondary substructure (as it appears in the crystal structure of the repressor-corepressor complex) to two successive major grooves of B-form DNA (Schevitz et al. 1985). Consistent with this, we have shown that the most critical operator determinants of repressor binding are organized into two 4-bp regions separated by a spacing of $4 \mathrm{bp}$, a geometry that positions functional groups of the base pairs in these two regions in successive major grooves on the same face of B-form DNA.

Our modeling of the binding of repressor to its operator suggests that two regions of Trp repressor dock closely with the operator: the hydrophilic surface of $\alpha$ helix D, including residues Gln-68, Arg-69, and Lys-72; and, the proximal end of $\alpha$-helix $\mathrm{E}$, including residues Ile-79, Ala-80, and Thr-81 (see Fig. 4 in Schevitz et al. $1985)$. We predict that side-chains of the residues in $\alpha$ helix D interact with base pairs more distal from the operator dyad, whereas $\alpha$-helix E side-chains contact more central base pairs. In particular, we suspect that the side-chains of Gln-68, Arg-69, or both, may donate a pair of hydrogen bonds to the $\mathrm{O}^{6}$ and $\mathrm{N}^{7}$ groups of the guanine of the $C: G$ base pair at position \pm 6 . This would account for our observation that a $C$ : G base pair is the only base pair that may be tolerated at this position. Second, a functional group on the side-chains of Arg-69 or Lys- 72 may donate a proton to the purine $\mathrm{N}^{7}$ at position \pm 5 , since we have shown that either a $\mathrm{T}: \mathrm{A}$ or $C: G$ base pair works at this position. Precedents for these specific interactions come from the models proposed for Cro binding (Ohlendorf et al. 1982; Hochschild and Ptashne 1986) and $\lambda$ repressor binding (Pabo and Lewis 1982; see also Pabo et al. 1983). Third, the Thr-81 hydroxyl group may form a hydrogen bond with the $\mathrm{O}^{4}$ group of thymine of the $\mathrm{A}$ : $\mathrm{T}$ base pair at position \pm 4 , since only $A: T$ and $C: G$ base pairs, which have congruent hydrogen bond donor-acceptor pairs in the major groove, work at this position. Such an interaction might result in a steric clash between the methyl group of Thr-81 and the methyl group of thymine when an A : T base pair is present at adjacent operator position \pm 3 , accounting for the observation that an $\mathrm{A}: \mathrm{T}$ base pair is the only base pair not tolerated at \pm 3 . Consistent with this, the mutant Thr- $81 \rightarrow$ Ser-81 repressor has an extended specificity of binding; it binds the operator with an $A: T$ base pair at \pm 3 , which wild-type repressor cannot (S. Bass, unpubl.).

Therefore, we predict that specific contacts between Trp repressor and its operator are made with both $\alpha$-helices D and E of the polypeptide. Although Trp repressor has a conserved helix-turn-helix secondary structure, it may use this structure to recognize DNA in a fundamentally different way than does $\lambda$ repressor, Cro protein, or 434 repressor, which predominantly use the second helix (Hochschild and Ptashne 1986; Anderson et al. 1987). Consistent with this idea, we find several $\operatorname{trp} R$ mutations that change amino acids Ile-79, Ala-80, and Thr-81 in $\alpha$-helix E to other residues, and alter the specificity of recognition of $t r p$ operators with changes at positions \pm 3 and \pm 4 . Not one of 24 different amino acid changes at these three residue positions affect recognition specificity at positions \pm 5 and \pm 6 , which we think are contacted by amino acids in $\alpha$-helix D. Kelley and Yanofsky (1986) failed to find dominant negative mutations that result in amino acid substitutions in $\alpha$-helix $\mathrm{D}$, since they used mutagens that preferentially cause $\mathrm{G}: \mathrm{C} \rightarrow \mathrm{A}: \mathrm{T}$ transitions, and the corresponding region of the $\operatorname{trp} R$ gene is particularly A : T-rich. Using site-directed mutagenesis, we have found more than 10 such changes. One of these mutant repressors, Lys- $72 \rightarrow$ Tyr, still binds the reference-type operator, whereas Lys- $72 \rightarrow$ Ala does not, arguing strongly that the sidechain of Lys- 72 makes a single, specific hydrogen bond with the operator (S. Bass, unpubl.).

The base pairs on either side of the four critical base pairs in each operator half-site (positions \pm 7 and \pm 2 ) do not appear to be involved directly in sequence-specific weak bond formation. However, it is clear from our results that changes in these base pairs can have mild but significant effects on binding (Table 1 and Fig. 4). We suspect that, as is the case for the 434 operator (Koudelka et al. 1987), changes at base pairs that are not directly involved in specific protein-DNA interactions may alter the local structure of trp operator DNA, and thereby affect the formation of weak bonds at neighboring positions.

\section{Genetic analysis of binding specificity}

We have shown that, using the challenge phage selection, we can identify the operator determinants of a DNA-binding interaction in a thorough manner. Challenge phages with variant trp operators that cannot be bound by the wild-type Trp repressor may be used to select or screen Trp repressor mutants with extended and 
altered specificities of binding. Further analyses of such mutants should allow us to test our hypotheses concerning the nature of the specific weak chemical bonds that underlie the Trp repressor-operator interaction.

\section{Materials and methods}

\section{Bacterial and phage strains}

Bacterial strains are derivatives of $S$. typhimurium LT2 or $E$. coli K12. Salmonella strains DB7000 (leuA-am414 sup ${ }^{0}$; Susskind et al. 1971) and its supE derivative MS1363 (Susskind 1980), used for the permissive growth of P22 arc-am phages, have been described. Otherwise isogenic hosts, MS1868 (sup ${ }^{0}$ ) and MS1883 (supE), also carry a mutation in the $h s d S B$ gene $\left(r^{-}\right)$ that inactivates one of three Salmonella restriction systems preventing efficient transformation (Grana et al. 1985). MS1582 is an immune $\left(\mathrm{c}^{+} \mathrm{mnt} \mathrm{t}^{+}\right)$lysogen of prophage P22 16amH1455 sieA44 Ap68tpfr49 in MS1363, used for the isolation of virulent mutants of P22 (Grana et al. 1985). The $\operatorname{trp} R:: \operatorname{Tn} 10$ insertion in strain TT2012 (LT2 trpR:: Tn10; from J. Roth) was moved into MS1582 by generalized transduction. Plasmids were constructed in E. coli strains JM101 (Messing et al. 1981), C600 (Appleyard 1954), or MM294 (Meselson and Yuan 1968) prior to transformation into Salmonella (Lederberg and Cohen 1974). The F'lac episome was introduced into MS1868 by conjugal transfer from E. coli $\mathrm{X} 90 / \mathrm{F}^{\prime} l a c I^{\mathrm{Q} 1}$ (Amann et al. 1983). Challenge phages were constructed by crosses between plasmids and phages (Youderian et al. 1983), and selected as described by Benson et al. (1986).

\section{Media, enzymes, and chemicals}

Media and general phage techniques have been described (Levine 1957; Levine and Curtiss 1961; Signer and Weil 1968; Susskind et al. 1971; Botstein et al. 1972; Weinstock et al. 1979; Youderian and Susskind 1980; Youderian et al. 1983).

Green tryptophan drop-out plates consist of M9 medium supplemented to $20 \mu \mathrm{g} / \mathrm{ml} \mathrm{L}$-arginine $\mathrm{HCl}$, L-aspartic acid, L-histidine $\mathrm{HCl}$, L-isoleucine, L-leucine, L-lysine $\mathrm{HCl}, \mathrm{L}$-methionine, L-phenylalanine, L-threonine, and L-tyrosine (Sigma); $0.06 \%$ alizarin yellow GG and $0.0067 \%$ aniline blue (Aldrich); and $0.8 \%$ glucose and $1.5 \%$ agar (Difco). Ampicillin and kanamycin (Sigma) were added to final concentrations of $50 \mu \mathrm{g} / \mathrm{ml}$. Plates were also supplemented with L-tryptophan and IPTG (Sigma) at various concentrations as described in the table and figure legends.

Restriction endonucleases, E. coli DNA polymerase I large fragment, T4 polynucleotide kinase, and T4 DNA ligase were purchased from New England Biolabs. Deoxyribonucleoside triphosphates and ATP were purchased from P-L Biochemicals. $[\alpha-32 \mathrm{P}] \mathrm{dATP}(700 \mathrm{Ci} / \mathrm{mmole})$, used in DNA sequence analyses, was from ICN. Synthetic trp operators were made on an Applied Biosystems Automated DNA Synthesizer (Model 381A) using phosphoramidite substrates (Caruthers 1982).

\section{Plasmids}

General procedures used in the construction, selection, and purification of plasmids have been described (Youderian et al. 1982). All plasmids are derivatives of pBR322 (Bolivar et al. 1977).

Plasmid pPY97 (Benson et al. 1986) has the EcoRII-HindIII fragment of P22 DNA carrying mnt, $\mathrm{P}_{\text {ant }}$ Omnt, and the proximal two-thirds of the arc gene inserted in the EcoRI-HindIII "backbone" of pZ150 (Zagursky and Berman 1984), a derivative of pBR322 carrying the phage M13 origin of replication and packaging initiation sites. Plasmid pPY97 and its derivatives thus create transcriptional fusions of Pant to the tet $A$ gene of pZ150, yet pPY97 is sensitive to tetracycline due to transcriptional repression by Mnt. Plasmid pPY140 is otherwise isogenic with pPY97, but carries a substitution of a synthetic stretch of DNA including a unique $X$ hol site for the Omnt region immediately $3^{\prime}$ to the ant promoter (Benson et al. 1986; see Fig. 2, below).

Plasmid pPY98 is otherwise isogenic with pPY97, except that it carries the backbone of pZ152 (Zagursky and Berman 1984). Plasmid pPY190, otherwise isogenic with pPY98, carries a substitution of a synthetic stretch of DNA including a unique SmaI site for the Omnt region immediately 3 ' relative to the ant promoter. pPY190 was derived from pPY 98 by site-directed mutagenesis of single-stranded plasmid DNA using a synthetic oligonucleotide $\quad\left(5^{\prime}\right.$-TCTACTATATTCCCGGGAATTCCGACCTGTATTGT-3'). The desired product was selected by its ability to confer increased tetracycline resistance upon a sensitive host, due to the substitution of synthetic DNA for Omnt, resulting in constitutive expression of the tetA gene.

The plasmid source of Trp repressor protein, pPY150, was constructed as described by Benson et al. (1986). It carries the M13 origin from plasmid pZ152 (Zagursky and Berman 1984) and a fusion of the lacUV5 promoter to the $\operatorname{trpR}$ structural gene from pRPG47 (Arvidson et al. 1986). Plasmid pPY158 is missing the smaller BamHI fragment of pPY150, which contains the entire $\operatorname{trp} R$ coding sequence.

\section{DNA sequence analyses}

Plasmid DNAs and double-stranded phage DNAs were sequenced by the method of Sanger et al. (1977), using an oligonucleotide complementary to the $5^{\prime}$ end of the arc gene as primer (5'-CGGCATTTTGCTCATTCC-3'). Single-stranded plasmid DNAs were prepared by the method of Zagursky and Berman (1984).

\section{Acknowledgments}

We thank Mimi Susskind and Charles Yanofsky for their detailed criticisms of the manuscript, and John Roth and Mimi Susskind for bacterial strains. This research was supported by National Institutes of Health grants GM 29456 to R.P.G. and GM34150 to P.Y., and by a UCLA Biomedical Research Grant to R.P.G. D.N.A. is supported by Cellular and Molecular Biology Training Grant GM 07185 from the National Institutes of Health.

\section{References}

Amann, E., J. Brosius, and M. Ptashne. 1983. Vectors bearing a hybrid trp-lac promoter useful for regulated expression of cloned genes in Escherichia coli. Gene 25: 167-178.

Anderson, J.E., M. Ptashne, and S.C. Harrison. 1987. Structure of the repressor-operator complex of bacteriophage 434. $\mathrm{Na}$ ture 326: 846-852.

Arvidson, D.N., C. Bruce, and R.P. Gunsalus. 1986. Interaction of the Escherichia coli Trp aporepressor with its ligand, Ltryptophan. J. Biol. Chem. 261: 238-243.

Appleyard, R.K. 1954. Segregation of new lysogenic types during growth of a doubly lysogenic strain derived from Escherichia coli K12. Genetics 39: 440-452.

Bennett, G.N. and C. Yanofsky. 1978. Sequence analysis of operator constitutive mutants of the tryptophan operon of Escherichia coli. J.Mol. Biol. 121: 179-192. 
Benson, N., P. Sugiono, S. Bass, L.V. Mendelman, and P. Youderian.1986. General selection for specific DNA-binding activities. Genetics 114: 1-14.

Bolivar, F., R.L. Rodriguez, M.C. Betlach, and H.W. Boyer. 1977. Construction and characterization of new cloning vehicles. I. Ampicillin-resistant derivatives of the plasmid pMB9. Gene 2: 75-93.

Botstein, D., R.K. Chan, and C.H. Waddell. 1972. Genetics of bacteriophage P22. II. Gene order and gene function. Virology 49: 268-282.

Caruthers, M.H. 1982. Chemical synthesis of oligodeoxyribonucleotides using the phosphite triester intermediates. In Chemical and enzymatic synthesis of gene fragments (ed. H.G. Gassen and A. Lang) pp. 71-79. Verlag Chemie, Weinheim, GDR.

Cohen, G.N. and F. Jacob. 1959. Sur la repression de la synthese intervenant dans la formation du tryptophan chez E. coli. C. R. Acad. Sci. Ser. D. 248: 3490-3492.

Grana, D., P. Youderian, and M.M. Susskind. 1985. Mutations that improve the ant promoter of Salmonella phage P22. Genetics 110: $1-16$.

Grove, C.L. and R.P. Gunsalus. 1987. Regulation of the $a r o H$ operon of Escherichia coli by the tryptophan repressor. $I$. Bacteriol. 169: 2158-2164.

Gunsalus, R.P. and C. Yanofsky. 1980. Nucleotide sequence and expression of Escherichia coli $\operatorname{trp} R$, the structural gene for the trp aporepressor. Proc. Natl. Acad. Sci. 77: 71177121.

Hochschild, A. and M. Ptashne. 1987. Homologous interactions of $\lambda$ repressor with $\lambda$ Cro and the $\lambda$ operator. Cell 44: 925933.

Joachimiak, A., R.L. Kelley, R.P. Gunsalus, C. Yanofsky, and P.B. Sigler. 1983. Purification and characterization of trp aporepressor. Proc. Natl. Acad. Sci. 80: 668-672.

Kelley, R.L. and C. Yanofsky. 1985. Mutational studies with the trp repressor of Escherichia coli support the helix-turnhelix model of repressor recognition of operator DNA. Proc. Natl. Acad. Sci. 82: 483-487.

Koudelka, G.B., S.C. Harrison, and M. Ptashne. 1987. Effect of noncontacted bases on the affinity of 434 operator for 434 repressor and Cro. Nature 326: 886-888.

Kumamoto, A., W. Miller, and R.P. Gunsalus. 1987. Escherichia coli tryptophan repressor binds multiple sites within the trp and aroH operators. Genes Dev. 1: 000-000.

Lederberg, E.M. and S.N. Cohen. 1974. Transformation of Salmonella typhimurium by plasmid deoxyribonucleic acid. J. Bacteriol. 119: 1072-1074.

Levine, M. 1957. Mutations in the temperate phage P22 and lysogeny in Salmonella. Virology 3: 22-41.

Levine, M. and R. Curtiss. 1961. Genetic fine structure of the C region and the linkage map of phage P22. Genetics 46: $1573-1580$.

Meselson, M. and R. Yuan. 1968. DNA restriction enzyme from E. coli. Nature 217: 1110-1114.

Messing, J., R. Crea, and P.H. Seeburg. 1981. A system for shotgun DNA sequencing. Nucleic Acids Res. 9: 309-321.

Ohlendorf, D.S., W.F. Anderson, R.G. Fisher, Y. Takeda, and B.W. Matthews. 1982. The molecular basis of DNA-protein recognition inferred from the structure of Cro repressor. $\mathrm{Na}$ ture 298: 718-723.

Pabo, C.O., S.R. Jordan, and A.D. Frankel. 1983. Systematic analysis of possible hydrogen bonds between amino acid side chains and B-form DNA. I. Biomole. Str. Dyns. 1: $1039-1049$.

Pabo, C.O. and M. Lewis. 1982. The operator-binding domain of $\lambda$ repressor: Structure and DNA recognition. Nature 298: $443-447$.
Pabo, C.O. and R.T. Sauer. 1984. Protein-DNA recognition. Annu. Rev. Biochem. 53: 293-321.

Sanger, F., S. Nicklen, and A. Coulson. 1977. DNA sequencing with chain terminating inhibitors. Proc. Natl. Acad. Sci. 74: 5463-5476.

Schevitz, R.W., Z. Otwinowski, A. Joachimiak, C.L. Lawson, and P.B. Sigler. 1985. The three-dimensional structure of $\operatorname{trp}$ repressor. Nature 317: 782-786.

Signer, E.R. and J. Weil. 1968. Recombination in bacteriophage $\lambda$. I. Mutants deficient in general recombination. I. Mol. Biol. 34: 261-271.

Susskind, M.M. 1980. A new gene of bacteriophage P22 which regulates synthesis of antirepressor. J. Mol. Biol. 138: 685713.

Susskind, M.M., A. Wright, and D. Botstein. 1971. Superinfection exclusion by P22 prophage in lysogens of Salmonella typhimurium. II. Genetic evidence for two exclusion systems. Virology 45: 638-652.

Weinstock, G., M.M. Susskind, and D. Botstein. 1979. Regional specificity of illegitimate recombination by the translocat able ampicillin-resistance element $\operatorname{Tn} 1$ in the genome of phage P22. Genetics 92: 685-710.

Yanofsky, C. 1971. Tryptophan biosynthesis in Escherichia coli. J. Am. Med. Assoc. 218: 1026-1035.

Youderian, P. S. Bouvier, and M.M. Susskind. 1982. Sequencedeterminants of promoter activity. Cell 30: 843-853.

Youderian, P. and M.M. Susskind. 1980. Bacteriophage P22 proteins specified by the region between genes 9 and erf. Virology 107: 270-282.

Youderian, P., A. Vershon, S. Bouvier, R.T. Sauer, and M.M. Susskind. 1983. Changing the DNA-binding specificity of a repressor. Cell 35: 777-783.

Zagursky, R.J. and M.L. Berman. 1984. Cloning vectors that yield high levels of single-stranded DNA for rapid sequencing. Gene 27: 183-191.

Zubay, G., D.E. Morse, W.J. Schrenk, and J.H.M. Miller. 1972. Detection and isolation of the repressor protein for the tryptophan operon of Escherichia coli. Proc. Natl. Acad. Sci. 69: $1100-1103$.

Zurawski, G., R.P. Gunsalus, K.D. Brown, and C. Yanofsky. 1981. Structure and regulation of $a r o H$, the structural gene for the tryptophan-repressible 3-deoxy-D-arabino heptulosonic acid-7-phosphate synthetase of Escherichia coli. I. Mol. Biol. 145: 47-73. 


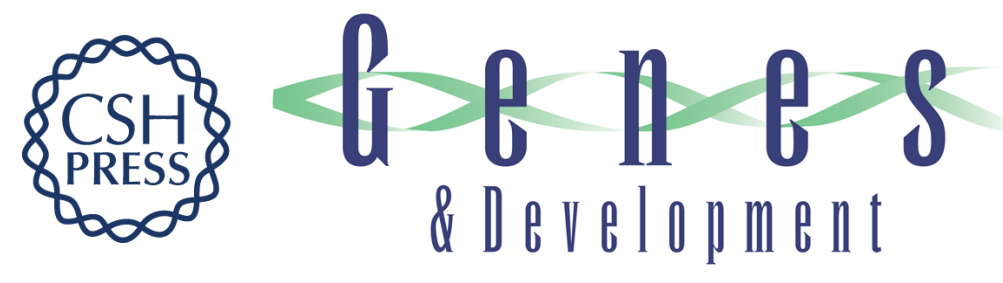

\section{DNA specificity determinants of Escherichia coli tryptophan repressor binding.}

S Bass, P Sugiono, D N Arvidson, et al.

Genes Dev. 1987, 1:

Access the most recent version at doi:10.1101/gad.1.6.565

References This article cites 39 articles, 13 of which can be accessed free at:

http://genesdev.cshlp.org/content/1/6/565.full.html\#ref-list-1

License

Email Alerting Receive free email alerts when new articles cite this article - sign up in the box at the top Service right corner of the article or click here.

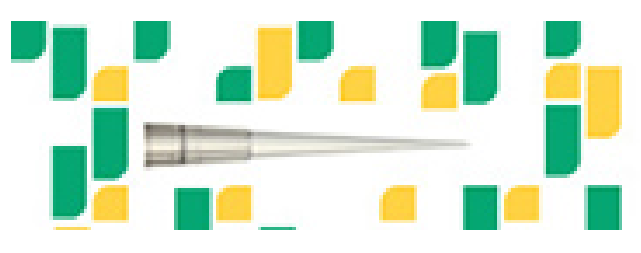

Focused on your science. 